\title{
Testing of a Reagent Kit "TEST-NRAS-TISSUE" to Determine Indications for Targeted Therapy in Patients
}

\author{
Andrey Toropovskiy ${ }^{1}$, Olga Pavlova ${ }^{1,2,3^{*}}$, Denis Viktorov ${ }^{1}$, Alexey Nikitin ${ }^{1}$ and Olga \\ Gulenko ${ }^{2}$
}

\author{
${ }^{1}$ TestGen LLC, Ulyanovsk, 432072, Russia \\ ${ }^{2}$ Samara State Transport University, Samara, 443066, Russia \\ ${ }^{3}$ Samara State Medical University, Samara, Russia \\ *Corresponding author. Email: casiopeya13@mail.ru
}

\begin{abstract}
The aim of the work was to determine the functional properties and efficiency of a reagent kit for determining the mutation status of the NRAS gene by allele-specific real-time PCR in human genomic DNA samples. These samples were taken from paraffin-fixed tissue samples (Test-NRAS-tissue) according to TU 21. 20.23-008-97638376-2016. It was introduced into clinical and laboratory practice to determine indications for target therapy with anti-EGFR monoclonal antibody-based drugs in patients diagnosed with colorectal cancer with the wild-type NRAS gene. In vitro, the diagnostic medical product "Reagent kit, used for determination of the NRAS gene mutation status by PCR$\mathrm{RV}$ in the human genomic DNA sample from paraffin-fixed tissue samples (Test-NRAS-tissue), was tested. During the clinical trials, 44 paraffin-fixed tissue samples (FFPE blocks) were analyzed in two series of experiments. That is, 88 clinical-laboratory experiments were performed, of which 46 experiments were made with genomic DNA samples with an established negative status of NRAS gene mutations and 42 experiments - with genomic DNA samples having an established positive status of NRAS gene mutations. The determination of the NRAS mutation status in the studied samples was performed using direct Sengler sequencing. Analysis and evaluation of the results of the clinical and laboratory tests of the Test-NRAS-tissue medical device confirmed that it allowed a qualitative determination of the mutation status of the twelfth codon (Gly12Asp, Gly12Cys, Gly12Ser), thirteenth codon (Gly13Asp, Gly13Arg) and sixty-first codon (Gln61Lys, Gln61Leu, Gln61Arg) of the NRAS gene. This was specified by allele-specific realtime PCR in human genomic DNA samples from paraffin-embedded tissue samples with high diagnostic sensitivity $(94.1 \%)$ and diagnostic specificity $(89.1 \%)$ at a $90 \%$ confidence level.
\end{abstract}

Keywords: reagent kit, TEST-NRAS-TISSUE, targeted therapy, DNA, diagnostic sensitivity, diagnostic specificity

\section{INTRODUCTION}

Colorectal cancer (CRC) occupies one of the leading positions in the structure of cancer morbidity, ranking third in the world after lung cancer and breast cancer. At the same time, there is an annual increase in the incidence of CRC [1]. The mortality rate from CRC is directly related to the frequency of distant metastasis. In Russia among patients with newly diagnosed colorectal cancer the share of patients with stage IV disease is $23.7-27.6 \%$. In other words, after removal of the primary tumor, metastases are detected in almost $50 \%$ of patients [2].

The basis of many cancers is dysregulation of the cell cycle, which leads to unrestrained cell growth and tumor formation. The causes of cell division disorders lie most often in somatic mutations - changes in the original genetic information of somatic cells. Disruption 
of gene expression and various chromosomal rearrangements (translocations, deletions, inversions and amplifications) can occur in the structure of protooncogenes, genes of growth factors, cell receptors and other biologically active genes. These abnormalities lead to loss of control over protooncogenes within the normal genome and trigger the processes of malignant transformation. Disturbances in the expression of proteins controlling the processes of apoptosis, proliferation and angiogenesis, epigenetic DNA changes of proteins controlling the vital activity of tumor cells affect the clinical course of the tumor process. It has been shown that differences in the expression of certain protein markers can explain why tumors comparable in stages, histological structures, and degrees of malignancy vary in the course of the disease [3].

One of the causes of colorectal malignancies can be mutations in the genes encoding the synthesis of RAS family proteins, which include HRAS, KRAS, NRAS, RRAS and other homologous proteins. RAS family proteins are involved in the activation of tyrosine kinase signaling pathways, which leads to gene mutations. For example, when EGFR (Epidermal Growth Factor Receptor) receptors are activated, signals are transmitted through the RAS-RAF-MEK-ERK multicomponent cascade. This process determines proliferative activity, differentiation ability, metastasis, escape from apoptosis, and induction of angiogenesis. Continuous activation of RAS leads to malignant cellular remodeling. The characteristic mechanism of overgrowth is point-activating mutations. The bestknown oncogenic mutations are mutations in the KRAS and NRAS genes with 12, 13 codons (2 exons), 59, 61 codons (3 exons), and 117, 146 codons (4 exons). KRAS, NRAS mutations lead to constant protein activation and mitotic signal transmission regardless of stimulation and drug inhibition of EGFR [4].

NRAS also belongs to the RAS oncogene family, whose gene is located on chromosome 1 [5]. NRAS differs from KRAS in the terminal part of the protein molecule, which determines its difference in transport, intracellular location, and function [6]. Mutation of the NRAS gene occurs in 3-5\% of cases of CRC, most commonly in codon 61 . KRAS and NRAS mutations are mutually exclusive $[6,7]$. The presence of a mutation in the NRAS gene determines resistance to anti-EGFR therapy for CRC [7-10].

Currently, monoclonal antibody-based targeted drugs, EGFR inhibitors cetuximab (cetuximab) and panitumumumab (panitumumab) are used to treat metastatic CRC. Binding of antibodies to EGFR leads to inhibition of invasion of tumor cells into normal tissues, preventing the spread of tumors to other organs. According to the May 15, 2014 letter No. 01I-692/14 "On new safety data for the drug Erbitux", mutations in the NRAS gene were added to the spectrum of mutations that must be investigated before prescribing Erbitux.

Currently, PCR amplification of the analyzed site followed by anomaly detection by various methods is used to detect mutations in colorectal tumors: sequencing, restriction fragment length polymorphism (RFLP) analysis, single-strand conformation polymorphism analysis (SSCP), hybridization with allele-specific oligonucleotides, high resolution melting (HRM), allele-specific real-time PCR (as-rv PCR) and others. Each of these methods has both advantages and disadvantages. Thus, the advantage of sequencing methods is their ability to detect all possible point mutations and their low cost. The limitation of sequencing is its insufficient sensitivity: 5-10\% of mutant alleles against wild-type DnA sequences for pyrosequencing and from 10 to $30 \%$ of mutant alleles for Sanger sequencing. The advantage of as-PCR is the presence of a closed system that protects from contamination and high sensitivity that detects up to $1 \%$ of mutant alleles against wild-type DNA. The disadvantages of as-rv PCR are the time-consuming optimization of allele-specific reactions and the ability to detect only selected mutations.

TestGen has developed "Reagent kit for determination of NRAS gene mutation status by PCRRV in human genomic DNA sample from paraffin-fixed tissue samples (Test-NRAS-tissue) according to TU 21.20. 23-008-97638376-2016" intended for professional use in medical organizations and clinical diagnostic laboratories of oncological profile to determine indications for targeted therapy with antiEGFR monoclonal antibody-based drugs in patients diagnosed with colorectal cancer.

The target analyte is the NRAS gene tested in patients diagnosed with colorectal cancer for qualitative determination of the mutation status of the twelfth codon (Gly12Asp, Gly12Cys, Gly12Ser), thirteenth codon (Gly13Asp, Gly13Arg) and sixty-first codon (Gln61Lys, Gln61Leu, Gln61Arg) of the NRAS gene.

Thus, the aim of our work was to determine the functional properties and efficiency of a reagent kit for determining the mutation status of the NRAS gene by allele-specific real-time PCR in human genomic DNA samples from paraffin-fixed tissue samples (TestNRAS-tissue). That was done according to TU 21. 20.23-008-97638376-2016 for its implementation in clinical and laboratory practice in order to determine the indications for target therapy with anti-EGFR monoclonal antibodies in patients diagnosed with colorectal cancer.

In order to realize the goal, the following tasks were solved:

- To study the reagent kit's compliance with its intended use; 
- Determine the effectiveness of a medical device for in vitro diagnostics in accordance with the intended use of the medical device by the manufacturer;

- Determine the quality of the reagent kit and the effectiveness of its use.

\section{Materials and Methods.}

Clinical trials of reagents using an in vitro diagnostic medical device "The set for determination of NRAS gene mutation status by PCR-RV in human genomic DNA sample from paraffin-fixed tissue samples (TestNRAS-tissue) were made according to TU 21.20.23008-97638376-2016" produced by TestGen LLC. They were conducted from July 18, 2018 to August 21, 2018 on the basis of the Federal State Budgetary Institution "Federal Scientific and Clinical Center for Specialized Types of Medical Care and Medical Technologies of the Federal Medical and Biological Agency" (FGBU FNKTs FMBA of Russia). That was done in accordance with the Order of the Russian Ministry of Health of January 9, 2014 No. 2n.

The total time of analysis using the Test-NRAStissue reagent kit is $2-2.5 \mathrm{~h}$ [11].

The analytical characteristics of the kit are presented in Table 1. A list of determinable mutations with mutation IDs is presented in Table 2.

Table 1. Analytical characteristics of the "Test-NRASTissue" reagent kit [11]

\begin{tabular}{|c|c|}
\hline $\begin{array}{l}\text { Analytical } \\
\text { specificity }\end{array}$ & $\begin{array}{l}\text { Specific to mutations in the twelfth } \\
\text { codon (Gly12Asp, Gly12Cys, } \\
\text { Gly12Ser), thirteenth codon } \\
\text { (Gly13Asp, Gly13Arg), and sixty-first } \\
\text { codon (GIn61Lys, Gln61Leu, } \\
\text { Gln61Arg) of the NRAS gene }\end{array}$ \\
\hline $\begin{array}{l}\text { Analytical } \\
\text { sensitivity }\end{array}$ & $\begin{array}{l}10 \text { copies of the NRAS gene in } 1 \mu l \\
\text { of DNA solution }\end{array}$ \\
\hline
\end{tabular}

Table 2. List of detectable mutations with mutation IDs

\begin{tabular}{|l|l|l|l|}
\hline $\begin{array}{l}\text { Set of mutations } \\
\text { determined with } \\
\text { the "Test-NRAS- } \\
\text { tissue" }\end{array}$ & $\begin{array}{l}\text { Changes in } \\
\text { nucleotides }\end{array}$ & $\begin{array}{l}\text { Changes } \\
\text { in amino } \\
\text { acids }\end{array}$ & $\begin{array}{l}\text { COSMIC } \\
\text { ID* }\end{array}$ \\
\hline Gly12Cys & c.34G>T & p.G12C & 562 \\
\hline Gly12Ser & c.34G>A & p.G12S & 563 \\
\hline Gly12Asp & c.35G>A & p.G12D & 564 \\
\hline Gly13Arg & c.37G >C & p.G13R & 569 \\
\hline Gly13Asp & c.38G >A & p.G13D & 573 \\
\hline Gln61Lys & c.181C>A & p.Q61K & 580 \\
\hline Gln61Leu & c.182A $>$ T & p.Q61L & 583 \\
\hline Gln61Arg & c.182A $>$ G & p.Q61R & 584 \\
\hline
\end{tabular}

* mutation identification number according to the international database of somatic mutations in cancer COSMIC (Catalog of Somatic Mutations in Cancer).

The specificity of the assay is determined by oligonucleotide primers selected for homologous gene segments. It is also determined by specific fluorescent oligonucleotide probes for hybridization with complementary sites of amplicons (specific amplification products), which excludes cross-reactions.

PCR mixtures of Gly12Asp, Gly12Cys, Gly12Ser, Gly13Asp, Gly13Arg, Gln61Lys, Gln61Leu, Gln61Arg are ready to use. They include PCR buffer (equimolar deoxynucleoside triphosphate (dNTP) mixture $600 \mathrm{nM}$, potassium chloride $-600 \mathrm{mM}$, Tris- $\mathrm{HCl} \mathrm{pH}-$ $8.8600 \mathrm{mM}$, glycerol - $0.6 \%$, Tween - $200.02 \%$, magnesium chloride $-30 \mathrm{mM}$ ). It was produced by Genterra, Russia, deionized water (reagent quality (type I) with a resistance of $18.2 \mathrm{MW}^{*} \mathrm{~cm}$ and TOC content less than $20 \mu \mathrm{g} / \mathrm{L}$ ), synthetic oligonucleotides (concentration $2.5 \mathrm{mM}$ each) and fluorescent probes (concentration $1 \mathrm{mM}$ ).

Taq polymerase, manufactured by Genterra, Russia, is ready-to-use and is a mixture of highly purified recombinant Taq DNA polymerase enzyme (enzyme concentration 5 units/ $\mu \mathrm{l}$ ) and specific monoclonal antibodies to polymerase.

The positive control sample (PCS) is ready-to-use and is a mixture of genomic DNA from human cell culture of the Jurkat line (manufactured by Thermo Fisher Scientific, USA) at a concentration of 400 copies of the NRAS gene in $1 \mu \mathrm{l}$ of DNA solution. And there was an artificially synthesized 300 bp insert, containing an equimolar mixture of Gly12Asp, Gly12Cys, Gly12Ser, Gly13Asp, Gly13Arg, Gln61Lys, Gln61Leu, and Gln61Arg mutations into the pAL-TA plasmid vector with a concentration of 20 plasmid DNA copies per $1 \mu \mathrm{l}$. It contains $5 \%$ mutant and $95 \%$ normal DNA copies.

Deionized water is used as a negative control sample.

All PCR mixtures contain primers and probes to an internal control sample (ICO). Probes to the ICO are labeled with HEX. This is a control for DNA extraction efficiency and the possible presence of inhibitors in the sample, the presence of which can lead to false-negative results. Passage of the reaction indicates sufficient nucleic acid extraction efficiency and the absence of PCR inhibitors.

Genomic DNA from human cell culture of the Jurkat line (manufactured by Thermo Fisher Scientific, USA) used to obtain the positive control sample and the control sample for sensitivity determination has negative NRAS gene status by mutations Gly12Asp, Gly12Cys, Gly12Ser, Gly13Asp, Gly13Arg, Gln61Lys, Gln61Leu, Gln61Arg. 
44 specimens of paraffin-fixed tissue from patients diagnosed with colorectal cancer were used as clinical trial subjects. Samples were obtained from a bank of residual aliquots of paraffin-fixed tissue (FFPE-blocks) formed in the course of routine therapeutic and diagnostic practice of FGBU FNKTs FMBA of Russia.

Inclusion criteria for the test subjects:

- Paraffin-fixed tissue samples from patients diagnosed with colorectal cancer.

\section{Exclusion Criteria:}

- The analyzed material must not be used if the storage and transportation conditions (temperature, duration, repeated freeze-thawing) are violated. The analyzed DNA must be stored at 2 to $8{ }^{\circ} \mathrm{C}$ and used for analysis within 24 hours. For storage over 24 hours, it is recommended to store the DNA solution at $-20^{\circ} \mathrm{C}$.

- The purity of the analyzed DNA, expressed in terms of the optical densities (A260/280nm) required for the assay, must be at least 1.4.

- The concentration of DNA sufficient for the assay must be $1-100 \mathrm{ng} / \mu \mathrm{l}$.

- Samples contaminated with the foreign biological material must not be used.

- Genomic DNA samples isolated from histologically confirmed tumor tissue must be used for analysis.

The number of samples is chosen based on the availability of samples in the residual tissue aliquots bank in the course of routine therapeutic and diagnostic practice of FGBU FNKTs FMBA of Russia.

For these patients, the mutation status of the NRAS gene was known and established on paraffin-embedded tissue samples prior to clinical trials of the registered medical device during the treatment and diagnostic process using Sanger sequencing of analyzed DNA samples. That was performed on an Applied Biosystems 3500 Dx genetic analyzer (sequencer), manufactured by Life Technologies Corporation, USA (No. FSZ 2011/09862 dated January 25, 2017), using the same primers as for forward and reverse PCR amplification for each amplicon.

No mutant DNA of the NRAS gene was detected in 23 samples provided by Sanger sequencing and was detected in 21 samples.

Two genomic DNA isolation procedures were performed from each tissue sample and analyzed using the Test-NRAS-tissue reagent kit in two series of experiments: In tissue samples numbered 1-24, the mutation status of the NRAS gene was established using Test-NRAS-tissue reagent kits LOT: 201806-65 and LOT: 201806-67, and in samples numbered 25-44 using
Test-NRAS-tissue reagent kits LOT: 201806-66 and LOT: 201806-68.

Extraction of human genomic DNA from tissue samples was performed using the Reagent kit for DNA extraction from biological material and determination of DNA fragmentation (IsoFrag-DNA-FFPE) according to TU 9398-001-7706721254-2011 (Proteinsintez, Russia, Registration certificate No. FSR 2011/12189 dated November 13, 2017).

Thus, 44 samples of paraffin-fixed tissue (FFPE blocks) were analyzed in two series of experiments during clinical trials, i.e., 88 clinical-laboratory experiments were performed. Among them, there were 46 experiments with genomic DNA samples with an established negative status of NRAS gene mutations and 42 experiments with genomic DNA samples with an established positive status of NRAS gene mutations.

Evidence of the correctness of the studied medical products was the coincidence of the results.

Qualitative determination of the mutation status of the twelfth codon (Gly12Asp, Gly12Cys, Gly12Ser), thirteenth codon (Gly13Asp, Gly13Arg) and sixty-first codon (Gln61Lys, Gln61Leu, Gln61Arg) of the NRAS gene by real-time allele-specific PCR in human genomic DNA samples from paraffin-fixed tissue samples involves three steps:

1) PCR preparation;

2) PCR amplification of DNA and real-time hybridization-fluorescence detection of amplification products;

3 ) interpretation of the results.

Amplification reactions are performed with human genomic DnA samples from paraffin-embedded tissue samples in the reaction buffer using primers specific to these DnA segments and the Taq polymerase enzyme. Allelic-specific fluorescence-labeled oligonucleotide probes are present in the reaction mixture for amplification, which hybridize with the complementary region of the amplified target DnA and are degraded by Taq polymerase, resulting in an increase in fluorescence intensity. This allows the accumulation of a specific amplification product to be recorded by measuring the intensity of the fluorescence signal. The detection of the fluorescence signal is performed directly during PCR using an amplifier with a "real-time" fluorescence detection system.

To perform PCR with "real-time" detection, we used a tablet-type amplifier DT-Prime (DnA-Technology, Russia).

Then we analyzed the curves of fluorescent signal accumulation by two channels: 
- the FAM channel registers a signal indicating accumulation of DNA amplification products of mutant variants of the NRAS gene.

- the HEX channel registers a signal indicating the accumulation of DNA amplification products from normal variants of the NRAS gene (acting as an internal control sample - ICO).

The results are interpreted based on the presence or absence of a threshold line crossing the fluorescence curve.

The results were interpreted based on the fluorescence signal level relative to the background in the corresponding channels for the control samples and DNA samples isolated from the samples under study. Interpretation was performed using the software of the used DT-Prime amplifier (DnA-Technology, Russia).

After the analysis of the samples and interpretation of the results with the provided reagent kit "Test-NRAStissue", the obtained data were compared with the results of Sanger sequencing of the analyzed DNA samples performed during the therapeutic and diagnostic process.

Evidence of the correctness of the studied medical devices was the coincidence of the results.

To calculate the diagnostic sensitivity and diagnostic specificity of the test kit used formulas given in paragraph 5.5 of GOST R 53022.3-2008 "Clinical laboratory technology. Quality requirements for clinical laboratory tests. Part 3. Rules for evaluation of clinical informativity of laboratory tests":

$$
\begin{aligned}
& \text { Sensitivity }=\frac{\text { True Positive }}{\text { True Positive }+ \text { False Negative }} \times 100 \% \\
& \text { Specificity }=\frac{\text { True Negative }}{\text { True Negative }+ \text { False Positive }} \times 100 \% .
\end{aligned}
$$

The results of the studies were evaluated according to Appendix $\mathrm{B}$ of the "Methodological recommendations on the order of examination of quality, effectiveness and safety of medical devices for state registration", approved by the Scientific-Expert Council of FGBU VNIIMT Roszdravnadzor on July 27, 2016.

Statistical processing of the study results was also performed.

\section{RESULTS}

When studying samples, each series of experiments is accompanied by staging the amplification with control samples. The result of PCR study is considered to be valid if the reactions "Gly12Asp", "Gly12Cys", "Gly12Ser", "Gly13Asp", "Gly13Arg", "Gln61Lys", "Gln61Leu" and "Gln61Arg" in tubes with the corresponding FAM and HEX channels pass no later than cycle 35. Correct results for OCO do not boost the fluorescence signal in reactions of "Gly12Asp", "Gly12Cys", "Gly12Ser", "Gly13Asp", "Gly13Arg", "Gln61Lys", "Gln61Leu" and "Gln61Arg" with FAM and HEX channels OCO.

Summary results of clinical tests of the submitted medical device samples compared with the Sanger sequencing results using the same primers as the forward and reverse PCR amplification for each amplicon are shown in Table 3.

\begin{tabular}{|c|c|c|c|c|c|c|c|}
\hline Sample & $\begin{array}{l}\text { "Test-NRAS } \\
\text { fabric", LOT: } \\
201806-65\end{array}$ & $\begin{array}{l}\text { "Test-NRAS } \\
\text { fabric", LOT: } \\
\text { 201806-66 }\end{array}$ & $\begin{array}{l}\text { Match/non- } \\
\text { match with } \\
\text { sequencing } \\
\text { result }\end{array}$ & $\begin{array}{l}\text { "Test-NAS } \\
\text { fabric", LOT: } \\
201806-67\end{array}$ & $\begin{array}{l}\text { "Test-NRAS } \\
\text { fabric", LOT: } \\
\text { 201806-68 }\end{array}$ & $\begin{array}{l}\text { Match/non- } \\
\text { match with } \\
\text { sequencing } \\
\text { result }\end{array}$ & $\begin{array}{l}\text { Mutation status } \\
\text { in the NRAS } \\
\text { gene as } \\
\text { determined by } \\
\text { Sanger } \\
\text { sequencing of } \\
\text { analyzed DNA } \\
\text { samples }\end{array}$ \\
\hline 1 & - & & coincidence & - & & coincidence & - \\
\hline 2 & Gln61Leu & & coincidence & Gln61Leu & & coincidence & Gln61Leu \\
\hline 3 & - & & coincidence & - & & coincidence & - \\
\hline 4 & Gln61Arg & & coincidence & Gln61Arg & & coincidence & Gln61Arg \\
\hline 5 & - & & coincidence & - & & coincidence & - \\
\hline 6 & Gln61Arg & & coincidence & Gln61Arg & & coincidence & Gln61Arg \\
\hline 7 & - & & coincidence & - & & coincidence & - \\
\hline 8 & - & & coincidence & - & & coincidence & - \\
\hline 9 & Gln61Lys & & coincidence & Gln61Lys & & coincidence & Gln61Lys \\
\hline 10 & - & & coincidence & - & & coincidence & - \\
\hline
\end{tabular}

Table 3. Summary results of clinical trials of the Test-NRAS-tissue reagent kit and Sanger sequencing 


\begin{tabular}{|c|c|c|c|c|c|c|c|}
\hline 11 & - & & coincidence & - & & coincidence & - \\
\hline 12 & Gly12Asp & & coincidence & Gly12Asp & & coincidence & Gly12Asp \\
\hline 13 & - & & coincidence & - & & coincidence & - \\
\hline 14 & - & & coincidence & - & & coincidence & - \\
\hline 15 & - & & coincidence & - & & coincidence & - \\
\hline 16 & - & & coincidence & - & & coincidence & - \\
\hline 17 & Gly12Asp & & coincidence & Gly12Asp & & coincidence & Gly12Asp \\
\hline 18 & Gln61Lys & & no match & Gln61Lys & & no match & - \\
\hline 19 & Gly13Arg & & coincidence & Gly13Arg & & coincidence & Gly13Arg \\
\hline 20 & - & & coincidence & - & & coincidence & - \\
\hline 21 & Gly12Ser & & coincidence & Gly12Ser & & coincidence & Gly12Ser \\
\hline 22 & Gln61Arg & & coincidence & Gln61Arg & & coincidence & Gln61Arg \\
\hline 23 & - & & coincidence & - & & coincidence & - \\
\hline 24 & - & & coincidence & - & & coincidence & - \\
\hline 25 & & Gln61Lys & coincidence & & Gln61Lys & coincidence & Gln61Lys \\
\hline 26 & & Gly12Cys & coincidence & & Gly12Cys & coincidence & Gly12Cys \\
\hline 27 & & - & coincidence & & - & coincidence & - \\
\hline 28 & & Gln61Leu & coincidence & & Gln61Leu & coincidence & Gln61Leu \\
\hline 29 & & - & coincidence & & - & coincidence & - \\
\hline 30 & & Gly12Asp & coincidence & & Gly12Asp & coincidence & Gly12Asp \\
\hline 31 & & - & coincidence & & - & coincidence & - \\
\hline 32 & & Gln61Arg & coincidence & & Gln61Arg & coincidence & Gln61Arg \\
\hline 33 & & Gln61Lys & coincidence & & Gln61Lys & coincidence & Gln61Lys \\
\hline 34 & & - & coincidence & & - & coincidence & - \\
\hline 35 & & Gln61Lys & coincidence & & Gln61Lys & coincidence & Gln61Lys \\
\hline 36 & & - & coincidence & & - & coincidence & - \\
\hline 37 & & Gly13Arg & coincidence & & Gly13Arg & coincidence & Gly13Arg \\
\hline 38 & & - & coincidence & & - & coincidence & - \\
\hline 39 & & - & coincidence & & - & coincidence & - \\
\hline 40 & & - & coincidence & & - & coincidence & - \\
\hline 41 & & Gln61Arg & coincidence & & Gln61Arg & coincidence & Gln61Arg \\
\hline 42 & & - & coincidence & & - & coincidence & - \\
\hline 43 & & Gln61Lys & coincidence & & Gln61Lys & coincidence & Gln61Lys \\
\hline 44 & & Gly12Cys & coincidence & & Gly12Cys & coincidence & Gly12Cys \\
\hline
\end{tabular}

During the clinical trial, 88 human genomic DNA samples were analyzed and 2 false positives were detected.

False positive results for sample \#18 in which the Gln61Lys mutation was detected using the Test-NRAStissue kit LOT: 201806-65 and LOT: 201806-67 can be explained by insufficient sensitivity of Sanger sequencing compared to the real-time allele-specific PCR method used in the Test-NRAS-tissue kit under study.

No false-negative results were detected in the clinical trials.

\section{DISCUSSION}

Thus, 44 samples of paraffin-fixed tissue (FFPE blocks) were analyzed in two series of experiments, i.e., 88 clinical and laboratory experiments were performed. Among them, there were 46 experiments with genomic DNA samples with an established negative status of the NRAS gene mutations and 42 experiments with genomic DNA samples with an established positive status of the NRAS gene mutations.

According to the results of clinical trials in a series of 42 experiments with genomic DNA samples with an 
established positive status of the presence of NRAS gene mutations, the registered medical product showed 42 true-positive results. No false-negative results were detected in the clinical trials.

In a series of 46 clinical trials with genomic DNA samples that were found to be negative for NRAS mutations, the registrable medical device showed 44 true negatives and 2 false positives. The detection of false positives may be due to insufficient sensitivity of Sanger sequencing compared to the allele-specific realtime PCR method used in the Test-NRAS-tissue kit under study.

According to the results of statistical processing of the obtained performance characteristics with $90 \%$ confidence probability, the diagnostic sensitivity of the studied medical device was $100 \%$, diagnostic specificity $-95.7 \%$.

According to the "Methodological recommendations on the order of examination of quality, effectiveness and safety of medical devices for state registration", approved by the Scientific-Expert Council of FGBU "VNIIIMT" Roszdravnadzor July 27, 2016, the result of $100 \%$ diagnostic sensitivity, obtained in 42 experiments with genomic DNA samples having the established positive mutation status of the NRAS gene can be considered as evidence for the effectiveness indicator diagnostic sensitivity of the medical device - at the level of 0.941 .

Also, the result of diagnostic specificity of $95.7 \%$ obtained in 46 experiments with genomic DNA samples with an established negative status of the presence of NRAS gene mutations can be considered as evidence for the performance indicator - diagnostic specificity of the medical device - at the level of $0.891(89.1 \%)$ with a $90 \%$ confidence probability.

Reproducibility of the results is $100 \%$.

Thus, the analysis and evaluation of the results of clinical and laboratory tests of the medical device confirmed the quality and effectiveness of the reagent kit.

\section{CONCLUSION}

As a result of clinical trials in the form of clinicallaboratory tests, it was found that the "Reagent kit for determining the mutation status of the NRAS gene by PCR-RV in human genomic DNA samples fixed in paraffin (Test-NRAS-tissue) according to TU 21.20.23008-97638376-2016" is clinically effective when used as prescribed by the manufacturer.

The product can be used in medical institutions and clinical diagnostic laboratories of the oncological profile. The scope of the reagent kit is clinical laboratory diagnosis, oncology, proctology.
Clinical trials of the medical device revealed the following advantages:

1. The manufacture produced modern, safe products, used in clinical laboratory conditions and in compliance with the requirements of the instructions for application.

2. Ease of use: the product is a set of reagents being ready to use.

3. The medical device allows the qualitative determination of the mutation status of the twelfth codon (Gly12Asp, Gly12Cys, Gly12Ser), thirteenth codon (Gly13Asp, Gly13Arg) and sixty-first codon (Gln61Lys, Gln61Leu, Gln61Arg) of the NRAS gene by allele-specific real-time PCR in the human genomic DNA sample from paraffin-embedded tissue samples. It has high diagnostic sensitivity $(94.1 \%)$ and diagnostic specificity $(89.1 \%)$ with a $90 \%$ confidence level.

4. Reproducibility of the $100 \%$ results confirms the high reliability of the kit.

\section{REFERENCES}

[1] W. De Roock, B. Claes, D. Bernasconi et al., Effects of KRAS, BRAF, NRAS and PIK3CA mutations on the efficacy of cetuximab plus chemotherapy in chemotherapy-refractory metastatic colorectal cancer: a retrospective consortium analysis, Lancet Oncol. 11 (2010) 753-762.

[2] S.A. Forbes, D. Beare, P. Gunasekaran et al., COSMIC: exploring the world's knowledge of somatic mutations in human cancer, Nuc. Acids Res. 43 (2015) D805-811. DOI: 10.1093/nar/gku1075

[3] W. De Roock, B. Claes, D. Bernasconi et al., Effects of KRAS, BRAF, NRAS and PIK3CA mutations on the efficacy of cetuximab plus chemotherapy in chemotherapy-refractory metastatic colorectal cancer: a retrospective consortium analysis, Lancet Oncol. 11 (2010) 753-762.

[4] R.J. Orton, O.E. Sturm, V. Vyshemirsky et al., Computational modelling of the receptor-tyrosinekinase-activated MAPK pathway, Biochem. J. 392(Pt 2) (2005) 249-261.

[5] M. Malumbres, M. Barbacid, RAS oncogenes: the first 30 years, Nat. Rev. Cancer. 3(6) (2003) 459-465. DOI: $10.1038 / \mathrm{nrc} 1097$

[6] K.M. Haigis, K.R. Kendall, Y. Wang et al., Differential effects of oncogenic K-Ras and N-Ras on proliferation, differentiation and tumor progression in the colon, Nat. Genet. 40 (2008) 600-608.

[7] G.A. Yanus, A.V. Belyaeva, A.O. Ivantsov et al., Pattern of clinically relevant mutations in 
consecutive series of Russian colorectal cancer patients, Med. Oncol. 30(3) (2013) 686. DOI: 10.1007/s12032-013-0686-5

[8] M.T. Seymour, S.R. Brown, S. Richman et al., Addition of panitumumab to irinotecan: results of PICCOLO, a randomized controlled trial in advanced colorectal cancer (aCRC), J. Clin. Oncol. 29(Suppl.) (2011) 3523.

[9] K. Oliner, M. Peeters, S. Siena et al., Evaluation of the gene mutations beyond KRAS as predictive biomarkers or response to panitumumab in a randomized, phase III monotherapy study of metastatic colorectal cancer (mCRC), J. Clin. Oncol. 29(Suppl.) (2011) 3530.

[10] J.Y. Douillard, T. Zemelka, G. Fountzilas et al., FOLFOX4 with cetuximab vs. UFOX with cetuximab as first-line therapy in metastatic colorectal cancer: The randomized phase II FUTURE study, Clin. Colorectal. Cancer. 13(1) (2014) 14-26.

[11] Instructions for using the "Reagent kit to determine the NRAS gene mutation status by PCR-RV in the human genomic DNA sample from paraffin-fixed tissue samples (Test-NRAS-tissue) according to TU 21.20.23-008-97638376-2016", version 2 of 27.03.20 